\title{
To Mathematical Modeling for Nonlinear Dynamics of Spacecraft Structures near-by the Disturbed Surface Using Hybrid Asymptotic Methods
}

\author{
Authors \\ S.I. Gomenjuk ${ }^{1}$, D.D. Gristchak ${ }^{2}$ \\ ${ }^{1}$ Professor of Mathematics Department, Zaporizhzhya National University, Zaporizhzhya, Ukraine \\ ${ }^{2}$ Postgraduate Student of Mathematics Department, Zaporizhzhya National University, Zaporizhzhya, \\ Ukraine \\ E-mail: gserega71@gmail.com
}

\begin{abstract}
In this paper an approximate analytical solution for mathematical model dynamic behavior of spacecraft (SC) which moves at the disturbed surface is discussed. A hybrid asymptotic approach on the basis of perturbation and phase-integral (or WKB) method have been employed to the singular nonlinear with timevariant periodic coefficients differential equation of motion in search of an approximate analytical solution. Key words: Nonlinear dynamics, spacecraft structure, disturbed surface, hybrid asymptotic approach, approximate analytical solution.
\end{abstract}

\section{INTRODUCTION}

Mathematical modeling for the nonlinear dynamics of structures near the disturbed surface is an important question for a variety of problems of SC systems. Of the interest, from the point of view of the dynamic effects appearance is the case of the coupled vibrations during the fly at the optional angle, for example, to the ocean wave surface [1-3]. It should be mentioned, that existing solutions, as a rule, are reduced to the solution of Mathieu-Hill equation on the conditions that the recuperate moment has non-linear (especially cube) character $[1,2]$ and dimensionless amplitude of the parametric excitation is a small quantity. However, in real conditions of SC system exploitation, value of this parameter can be not small, that can leads to significant lost quality of dynamic characteristics. Most interest from the point of view of origin of different dynamic effects presents the case of joint vibrations of vehicle in plane of heel and in plane of tangage. The mechanical analogue of this process can serve the model of the mathematical pendulum vibration in a vertical plane with the hesitating on the set law supporting point. This paper continues the discussions of the publications in this sphere and deals with the discussion of proposed an approximate analytical solution of the problem.

\section{SINGULAR NONLINEAR DIFFERENTIAL EQUATION OF THE PROBLEM. HYBRID ASYMTOTIC SOLUTION}

Mathematical model for dynamic problem of the spacecraft-absorber system is presented on Fig. 1.

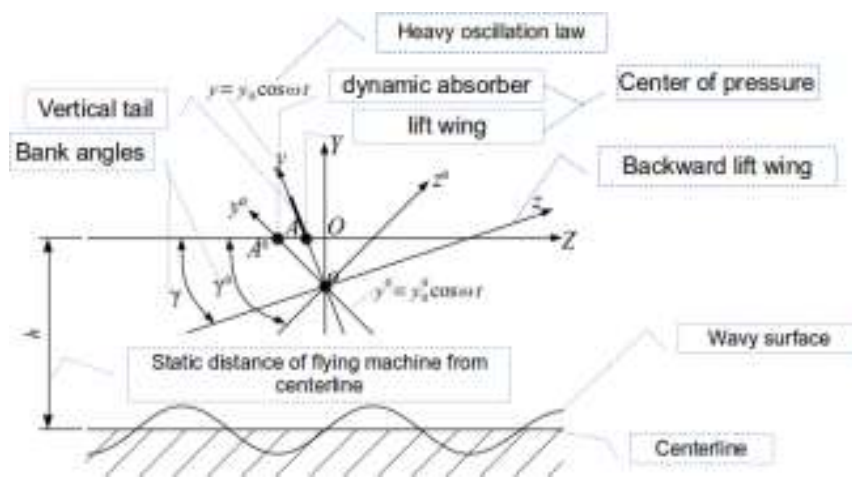

Fig. 1 - Calculation chart « $\mathrm{SC}$ - absorber» Differential equation system, describing of «SC dynamic absorber» vibration process is as follows [2]: 


$$
\begin{aligned}
& I_{x} \frac{d^{2} \gamma}{d t^{2}}+n \frac{d \gamma}{d t}+C_{11}(t) \gamma+C_{22}(t) \gamma^{m}+I_{x^{0}}\left(y+y^{0}\right)=0, \\
& I_{x^{0}}\left(y+y^{0}\right)+n_{0} \gamma^{0}(t)+C_{1}(t) y^{0}(t)=0 .
\end{aligned}
$$

With due regard to the second equation of the system (1) the main differential equation of tangage motion acquires the form

$$
I_{x} \ddot{\gamma}(t)+n_{0} \dot{\gamma}(t)+C_{11} \gamma(t)+C_{22} \gamma^{m}(t)=F\left(\gamma^{0}, t\right)
$$

where

$$
\begin{aligned}
& F\left(\gamma^{0}, t\right)=n_{0} \gamma^{0}(t)+C^{0} \gamma^{0}(t), \\
& n=\frac{1}{1+\frac{y_{0}}{h} \cos (\omega t)}\left(n_{1}+n_{2}\right)+n_{3}+n_{0} .
\end{aligned}
$$

$n_{0}$ - coefficient belonging to the dynamic absorber; $n_{1}-$ to lift wing; $n_{2}-$ to stabilizator; $n_{3}-$ to vertical fin.

Therefore, the problem with attached dynamic absorber reduces itself to the right part $F\left(\gamma^{0}, t\right)$ of initial equation (2).

In such a case calculation chart takes the following form [2] presented in Fig.1. In given Fig.1 the frame of axis $y^{0} o z^{0}$ is related to the dynamic absorber.

Equation (2) can be presented in the form:

$$
\varepsilon^{2} \ddot{\gamma}(t)+B_{1}(t) \gamma(t)+\eta \gamma(\tau)^{m}=F\left(\gamma^{0}, t\right),
$$

where $\varepsilon$ and $\eta$ are asymptotic parameters.

Note, that for particular case, when $m=3$, differential equation (4) corresponds to Matieu equation. Here it will be discussed general case for the parameter nonlinearity $m$.

Using hybrid approach on the basis of perturbation technique and two - terms approximation of phaseintegral method [4], an approximate analytical solution of equation problem (4) is:

$$
\begin{aligned}
& \gamma(\tau)=\frac{1}{B_{1}^{\frac{1}{4}}(\tau)}\left[\operatorname{Sin} K(\tau)\left(c_{1}+\bar{c}_{1}(\tau)+\eta \bar{d}_{1}(\tau)\right)+\right. \\
& \left.+\operatorname{Cos} K(\tau)\left(c_{2}+\bar{c}_{2}(\tau)+\eta \bar{d}_{2}(\tau)\right)\right]
\end{aligned}
$$

where first term in (5) corresponds to linear homogeneous solution, second term - to influence of right side of equation (4), third term - to non linearity influence.

Solution (5) can be rewrite as

$$
\begin{aligned}
& \gamma(t)=\operatorname{Exp}\left[-\frac{v(\tau)}{2}\right]\left\{\operatorname { S i n } K ( \tau ) \left[s_{1}+\right.\right. \\
& +\varepsilon \int \frac{F\left(\gamma^{0}, t\right)+\eta\left[\gamma_{0}(\tau)\right]^{m}}{B_{1}(\tau)^{0.25}} \operatorname{Cos} K(\tau) d \tau+ \\
& \operatorname{CosK}(\tau)\left[s_{2}-\varepsilon \int \frac{F\left(\gamma^{0}, t\right)+\eta\left[\gamma_{0}(\tau)\right]^{m}}{B_{1}(\tau)^{0.25}} \operatorname{Sin} K(\tau) d \tau\right\}
\end{aligned}
$$

where non dimensional parameters and solution functions are:

$$
\gamma_{0}^{0}(\tau)=\frac{\operatorname{Exp}\left[-\frac{v(\tau)}{2}\right]}{B_{1}^{\frac{1}{4}}(\tau)}\left[c_{1} \operatorname{Sin} K(\tau)+c_{2} \operatorname{Cos} K(\tau)\right],
$$

$t=\frac{2 \tau}{\omega}, \quad \mu=\frac{a y_{0}}{h}=k a, \varepsilon^{2}=\frac{1}{a}, a=\frac{4 c_{11}}{I_{x} \omega^{2}}, \quad v=\frac{2 n}{I_{x} \omega^{2}}$,

$\eta=\frac{4 c_{22}}{I_{x} \omega^{2}}, N(\tau)=\left[\gamma_{0}(\tau)\right]^{m}$,

$\bar{c}_{1}(t)=\int \frac{F\left(\gamma^{0}, t\right) y_{2}^{0}(t)}{-W(t)} d t, \bar{c}_{2}(t)=-\int \frac{F\left(\gamma^{0}, t\right) y_{1}^{0}(t)}{-W(t)} d t$,

$\gamma_{1}^{0}(t)=\frac{\operatorname{Exp}\left[-\frac{v(\tau)}{2}\right] \operatorname{Sin} K(\tau)}{B_{1}(\tau)^{0.25}}$,

$\gamma_{2}^{0}(t)=\frac{\operatorname{Exp}\left[-\frac{v(\tau)}{2}\right] \operatorname{Cos} K(\tau)}{B_{1}(\tau)^{0.25}}$,

$W(\tau)=\frac{\operatorname{Exp}\left[-\frac{v(\tau)}{2}\right] K^{\prime}(\tau)}{-B_{1}^{0.5}(\tau)}$,

$\bar{c}_{1}(\tau)=\varepsilon \int \frac{F\left(\gamma^{0}, \tau\right) \operatorname{Exp}\left[-\frac{\nu(\tau)}{2}\right] \operatorname{Cos} K(\tau)}{B_{1}^{0.5}(\tau)} d \tau$,

$\bar{c}_{2}(\tau)=-\varepsilon \int \frac{F\left(\gamma^{0}, \tau\right) \operatorname{Exp}\left[-\frac{v(\tau)}{2}\right] \operatorname{Sin} K(\tau)}{B_{1}^{0.5}(\tau)} d \tau$,

$\bar{d}_{1}(\tau)=\varepsilon \int \frac{\eta N\left(\gamma^{0}, \tau\right) \operatorname{Cos} K(\tau)}{B_{1}^{0.5}(\tau)} d \tau$

$\bar{d}_{2}(\tau)=-\varepsilon \int \frac{\eta N\left(\gamma^{0}, \tau\right) \operatorname{Cos} K(\tau)}{B_{1}^{0.5}(\tau)} d \tau$

$B_{1}(\tau)=1-2 \frac{\mu}{a} \operatorname{Cos} 2 \tau-\frac{v(\tau)^{2}}{4 a}-\frac{\dot{\nu}(\tau)}{2 a}$,

$K(\tau)=\int \varepsilon^{-1} B_{1}^{\frac{1}{2}}(\tau) d \tau$,

$c_{11}(\tau)=\frac{1}{\left(\frac{h}{y_{0}}+\cos \omega \tau\right)+\left(1+\frac{y_{0}}{h} \cos \omega \tau\right)}\left(C_{1}+C_{2}\right)$, 


$$
c_{22}(\tau)=\frac{1}{\left(\frac{h}{y_{0}}+\cos \omega \tau\right)\left(1+\frac{y_{0}}{h} \cos \omega \tau\right)}\left(3 C_{1} \frac{l_{1}^{2}}{7 h_{1}^{2}}+3 C_{2} \frac{l_{2}^{2}}{7 h_{2}^{2}}\right),
$$

where $C_{1}, C_{2}$ - have to be obtained from [1]. $c_{1}, c_{2}-$ are constants, determined from the given initial conditions.

For the given parameters of SC and initial conditions

$$
\begin{aligned}
& \gamma(0)=\gamma_{0}, \\
& \gamma^{\prime}(0)=0,
\end{aligned}
$$

expression (5) is an approximate analytical solution problem as function from the amplitude of periodic in time damping coefficient $n$ and parameter $\frac{y_{0}}{h}$.

\section{IFLUENCE OF DEPENDING ON TIME PARAMETERS ON FREE VIBRATIONS OF SYSTEM}

It is necessary to note, that for homogeneous problem, when $F\left(\gamma^{0}, t\right)=0$, linear initial equation becomes

$$
\varepsilon^{2} \ddot{\gamma}(t)+\left[a^{*}(\tau)-2 \mu \operatorname{Cos} 2 \tau\right] \gamma(t)=0,
$$

where $a^{*}(\tau)=a-\frac{v^{2}(\tau)}{4}-\frac{v^{\prime}(\tau)}{2}$, and basic zone of SC system instability is function on dependent from time damping coefficient and vertical axis of Aines - Strett diagram removes in the left side [1].

For the given parameters SC system $v(\tau)=v_{0}(1+0.1 \tau), a=100, \varepsilon=0.1, v_{0}=1, \frac{\mu}{a}=0.1$ and initial conditions (17) an analytical solution of equation (18) in one - term WKB approximation is:

$$
\begin{aligned}
& \gamma(\tau)=\operatorname{Exp}\left[-\frac{(1+0.1 \tau)}{2}\right]\{0.0092 \operatorname{Sin}[8.94 \tau+ \\
& \left.\left.+0.747 \tau^{3}\right]+1.6487 \operatorname{Cos}\left[8.94 \tau+0.747 \tau^{3}\right]\right\}
\end{aligned}
$$

Results of numerical calculations are given in Fig.2.

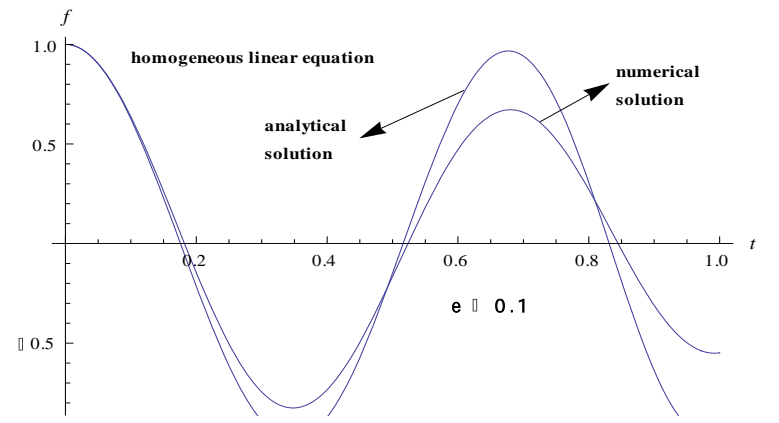

Fig.2 Comparison of numerical and analytical solutions

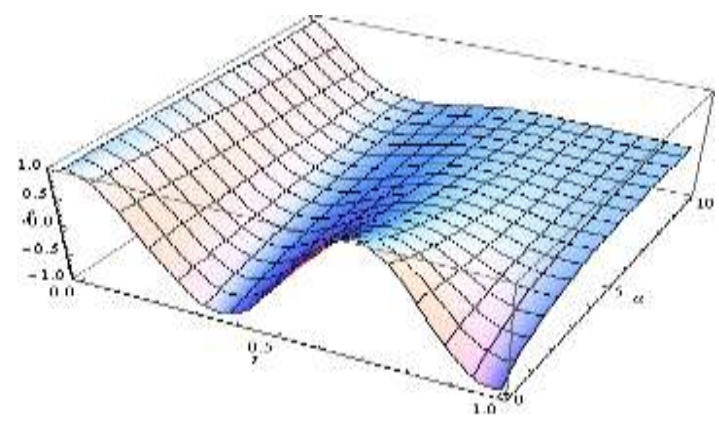

Fig.3 Influence of damping parameter $a$ at $v_{0}=1$, $v(\tau)=v_{0}(1+a \tau)$

\section{CONCLUSIONS}

Approximate analytical solutions for nonlinear dynamics of vehicle offers near-by the disturbed surface in the case when dimensionless amplitude of parametric excitation $\mu$ is being function of time is suggested. Proposed hybrid asymptotic approach can be efficient for further investigations of dynamic stability of structure near the wavy surface. The special attention in further researches must be directionally on the large values of parameter $\mu$ For this problem can be effective hybrid (WKB-G) asymptotic approach with the use of Galerkin's orthogonality principle.

\section{REFERENCES}

1. V.V. Olkov, I.N. Gusev, Dynamic stability of aircraft near-by the disturbed surface, in book "Perturbation methods in mechanics", Novosibirsk, "Nauka", 1982, p. 105-111.

2. I.N. Gusev, Transient behaviors of motion of aircraft in plane heel, in book "Perturbation methods in mechanics", Novosibirsk, "Nauka", 1979, p. 171-180.

3. V.Z. Gristchak, V.N. Kabak, Double Asymptotic Method for Nonlinear Forced Oscillations Problems of Mechanical Systems with Time Dependent Parameters, Technische Mechanik. - 1996. - Band 16, Heft 4. - P. 285-296.

4. V.Z. Gristchak, J.A. Ganilova, Application of a Hybrid WKB-Galerkin Method in Control of the Dynamic Instability of a Piezolaminated Imperfect Column, 
Technische Mechanik. - 2006. - №26(2). P. $106-116$.

5. V.A. Azarscov, D.V. Gristchak, D.D. Gristchak, Oscillation Damping of AirSpace Structures With Joint-Up Dynamic Absorber, Proceedings The Sixth World Congress "Safety in Aviation and Space Technologies", September 23-25, 2013, Kiev, Ukraine.

6. D.D. Gristchak, Vibration of spacecraft structure with joint-up dynamic absorber and periodic damping coefficient near disturbed surface, Problems of computational mechanics and strength of structures, 24, 2015, p.286-294. 